\title{
Personality traits of parents and problems behaviors of the children with intellectual disabilities
}

\author{
Dr. Ashutosh Kumar Santosh ${ }^{1 *}$, Jai Prakash ${ }^{2}$
}

\section{ABSTRACT}

Background: The children with Intellectual Disabilities (ID) are known to exhibit different types of problem behaviors and significant limitations in their day to day age appropriate activities and adaptive behaviors. All parents of children with ID are not alike. They hold different personality traits, which are responsible for their behavioral expression. Therefore personality traits of the parents of children with ID have significant impact on the development of problem behavior of their children, Aims and objective: Hence the present study was conducted with the sole purpose of (1) to examine the types of problem behavior shown by the children with ID having different categories of ID and (2) to identify the types of the personality traits holds by the parents of children with different categories of ID (3) to examine the relationship between the personality traits of the parents and expression of problem behaviors of the children with ID. Hypothesis: - There is no significant difference in Problem Behaviors exhibited by children with different levels of ID. - There is no significant difference in Personality traits of parents having children with different levels of ID. Methodology: Sample: Sample consists of 45 parents (either father or mother) who have children with mild, moderate or severe level of ID and must exhibit the problem behaviors. Equal numbers of parents were chosen in all three groups through purposive sampling techniques. Research design: This is the cross sectional study where the impact of different types of problem behaviors exhibited by the children having different categories of ID was examined over the different personality traits of the parents. Variables: The independent variables are the different types of problem behaviors exhibited by the children with ID having different categories of ID and the dependent variable is the personality traits of the parents having children with ID. Research tools: Socio-demographic data were collected on the revised version of the NIMH Socio Economic Status (SES) Scale (NIMH, 1999). Problem behaviors were assessed by using Behavioral Assessment Scales for Indian Children with Mental Retardation (BASIC-MR) Part- B developed by Peshawaria, \& Venkatesan, 1992. It measures ten types of problem behavior commonly found in children with ID. Personality traits of the parents were assessed by using Sixteen Personality Factors (16PF) questionnaire, developed by Cattell, 1967 \&Kapoor, 1978. Statistical Analysis of data: Obtained data were analyzed by using Chi-square test and F-test. Software SPSS version 17.0 was used for statistical treatment of data. Result: Result indicates that violent and destructive behavior (F

\footnotetext{
${ }^{1}$ Assistant Professor Clinical Psychology, Advanced Neuro Hospital, Boring Road, Patna (Bihar), India ${ }^{2}$ Assistant Professor, Department of Psychology, A.N.S College, Nabinagar, Aurangabad, Bihar, India *Responding Author

Received: June 05, 2020; Revision Received: June 22, 2020; Accepted: September 25, 2020
}

(C) 2020, A K Santosh \& J Prakash; licensee IJIP. This is an Open Access Research distributed under the terms of the Creative Commons Attribution License (www.creativecommons.org/licenses/by/2.0), which permits unrestricted use, distribution, and reproduction in any Medium, provided the original work is properly cited. 
= 13.17), self-injurious behavior $(\mathrm{F}=10.41)$, and repetitive behavior (6.69) was found significant on 0.01 levels, whereas temper tantrum behavior $(\mathrm{F}=4.67)$ and rebellious behavior $(\mathrm{F}=3.62)$ was also found significant at 0.05 levels. These five types of problem behavior is present in ascending order in all three groups of children with ID (Mild, moderate and severe levels of ID). These problem behaviors are closely related to the parent's personality traits of I (Sensitivity) (chi-square $=9.82$, significant at 0.05 level) and Q1 (Openness to change) (chi-square $=11.58$, significant at 0.05 level). Children of such parents were involving more in problem behavior. Those problem behaviors which was not found significant were misbehavior with others $(\mathrm{F}=1.88)$, odd behavior $(\mathrm{F}=0.46)$, hyperactive behavior $(\mathrm{F}=0.48)$, antisocial behavior $(\mathrm{F}=2.33)$, and fears $(\mathrm{F}=2.25)$.

Keywords: Personality traits, problem behaviors, intellectual disability, adaptive behaviors, parents of children with ID.

The term Intellectual Disability (ID) is relatively new which appears in the Right of Persons with Disabilities Act, 2016 (RPWD Act, 2016) and published in the Gazette of Government of India.

ID is considered as a developmental disability evident in childhood as significantly belowaverage intellectual and adaptive functioning. People with ID experience difficulties with day-to-day activities to an extent that reflects both the severity of their cognitive deficits and the type and amount of assistance they receive. (Barlow \& Durand, 2009, p. 518).

According to the Diagnostic and statistical manual of Mental Diseases $-4^{\text {th }}$ edition, 1994 (DSM-4), the ID which was earlier known by the name of mental retardation, was defined as A. Significantly sub-average intellectual functioning: an IQ of approximately 70 or below on an individually administered IQ test.

B. Concurrent deficits or impairments in present adaptive functioning in at least two of the following areas: communication, self-care, home living, social/interpersonal skills, use of community resources, self-direction, functional academic skills, work, leisure, health, and safety.

RPWD Act, 2016 define ID as: Intellectual disability, a condition characterized by significant limitation both in intellectual functioning (reasoning, learning, problem solving) and in adaptive behavior which covers a range of every day, social and practical skills.

As per DSM - 4, there are four types of ID: Mild ID - those who have IQ level of in the range of 50 to 70, moderate ID: IQ in the range of 35 to 49, Severe ID: IQ in the range of 20 to 34 and profound ID: having IQ less than 20 on any standardized test of intelligence. The manifestations of ID are varied. Some individuals function quite well. Individuals with mild or moderate levels of impairments might be able to use public transportation independently, hold jobs, and manage money. Others, however, have significant cognitive and physical impairments and require considerable assistance to carry on day-to-day activities. Severe and profound levels of ID may need help to eat and bathe and to do activities of day to day life.

Whatever the category of limitations they may have (mild, moderate, severe, profound levels of ID), they are known to exhibit different types of problem behaviors. Rastogi (1984) found that children with ID having lower concentration, showing temper tantrums, hyperactive, feeling of misery and disturbed peer relations. Shrivastava et al., 1981 found that self-injury 
behavior, aggression, property destruction, eating inedible things, nail biting, self-talking, self-laughing, wandering etc. were more common in the children with ID.

There are several reasons which are lying behind the problem behaviors such as genetic make-up, psychological factors, sociological factors and environmental factors that perpetuate to indulge in the problem behaviors. Apart from that, parental behavior and their unique response towards the children behavior occupied much prime importance than any other factors. Its play a major role in determining the types of problem behaviors exhibited by the children with ID. (Burchinal, 1964, Mc Cathy, 1968, Paulous, 1969, Peniston, 1976; Glassner and Navarse, 1965).

The behaviors of the individuals are determined by their Personality traits. A personality trait is defined as a dynamic organization, inside the person, of psychophysical systems that create a person's characteristic pattern of behavior, thoughts and feelings (Carver and Scheier 2000). Diagnostic and Statistical Manual of the Mental Diseases $-4^{\text {th }}$ edition, 2000 (DSM - 4) which was published by American Psychiatric Association, defined personality traits as a enduring patterns of perceiving, relating to, and thinking about the environment and oneself that are exhibited in a wide range of social and personal contexts. According to Belsky's model, parental personality trait was considered as the theoretically most influential determinant of parenting because it is thought to affect parental behavior both directly and indirectly.

Lisa et al, (2001) suggested that mother's warm, supportive relationships with child, has impact on child's internalizing problems, externalizing problems, and social competence in community context. Therefore, personality traits of parents have significant impact on development of problem behavior of children with ID.

\section{Aims and objectives}

Hence, the present study was conducted with the sole purpose of:

1. To examine the types of problem behavior shown by the children with ID having different categories of ID.

2. To identify the types of the personality traits holds by the parents of children with different categories of ID.

3. To examine the relationship between the personality traits of the parents and expression of problem behaviors of the children with ID.

\section{Hypothesis}

To examine its aims and objectives, following hypothesis were formulated:

1. There is no significant difference in Problem Behaviors exhibited by children with different levels of ID.

2. There is no significant difference in Personality traits of parents having children with different levels of ID.

\section{RESEARCH METHODOLOGY}

\section{Sample size}

Sample consists of 45 parents (either father or mother) who have children with mild, moderate or severe level of ID and must exhibit the problem behaviors. Equal numbers of parents were chosen in all three groups through purposive sampling techniques.

\section{Inclusion/exclusion criteria:}

(c) The International Journal of Indian Psychology, ISSN 2348-5396 (e)| ISSN: 2349-3429 (p) | 3 
Only those were chosen in this present study who have given their consent to be a part of this study, who have children with ID (mild, moderate or severe levels of ID) and must exhibiting the problem behaviors. Those were excluded from the study who could not read and write, having children with other types of disabilities such as such as learning disabilities, cerebral palsy, physically handicapped, hearing impairment, visual impairment and epilepsy.

Research design: This is the cross-sectional study where the impact of different types of problem behaviors exhibited by the children with ID having different categories of ID was examined over the different personality traits of the parents.

Variables: The independent variables are the different types of problem behaviors exhibited by the children having different categories of ID and the dependent variable is the personality traits of the parents having children with ID.

\section{Research tools}

Socio-demographic details A revised version of the NIMH Socio Economic Status (SES) Scale (NIMH, 1999) was employed to collect the data related to socio-demographic details of the sample. This scale offers numerical points of different ranges for computation of a SES score/ level for an individual by taking into account details such as parental occupation and education, annual family income, combined properties as well as per capital income of the family. Higher points on the scale indicate better socio economic status level.

Behavioral Assessment Scales for Indian Children with Mental Retardation (BASICMR) Part- B: Problem behaviors were assessed by using Behavioral Assessment Scales for Indian Children with Mental Retardation (BASIC-MR) Part- B developed by Peshawaria, \& Venkatesan, 1992. This scale is divided into two parts: Part A is for adaptive behavior contains seven domains with 280 items, and Part B is for problematic/maladaptive behavior, it contains 10 domains with 75 items all together. The ten domains are violent and destructive behaviors, temper tantrums, misbehaviors with others, self-injurious behaviors, repetitive behaviors, odd behaviors, hyperactive behaviors, rebellious behaviors, antisocial behaviors and fears. The range of score under problem behaviors is $0-2$ where 0 indicates problem behaviors not occurring at all, score 1 indicates that problem behavior is occurring sometimes, and score 2 indicates that problem behavior is occurring most frequently. The maximum possible score on BASIC - MR (Part - B) is 150 (75 items x 2 maximum possible score). To determine the severity of problem behavior, it is recommended in the test manual to calculate the score in the percentage by using the formula of total raw score/divided by total maximum possible score i. e. 150 x multiple with 100 . Lower score indicates less problem behavior.

The information on the scale is collected through direct observation of the child and by interviewing parents. The scale is exclusively developed for the assessment and program planning for the persons with mental retardation.

16 PF Questionnaires: Personality traits of the parents of children with ID were assessed by using Sixteen Personality Factors (16PF) questionnaire, developed by Cattell, 1967. Hindi version of this test was developed by Kapoor, 1978. It is a self- reported personality test that assesses 16 types of personality traits. Each person contains all of these 16 traits to a certain degree, but they might be high in some traits and low in others. Form - A of 16 PF questionnaires was used in this study. The questionnaire contains 187 questions under 16

(c) The International Journal of Indian Psychology, ISSN 2348-5396 (e)| ISSN: 2349-3429 (p) | 4 


\section{Personality Traits of Parents and Problems Behaviors of the Children with Intellectual Disabilities}

domains of personality traits. Depending up on the educational background and comfort ability of the parents in the language use, both English and Hindi version of the questionnaire was used to assess their personality traits.

Individually administration of the test took about 35-50 minutes for the paper-and-pencil version. The test was hand-scored using a set of scoring keys. Scores on the 16PF are presented on a 10-point "sten" scale, or standard-ten scale. The sten scale has a mean of 5.5 and a standard deviation of 2, with scores below 4 considered low and scores above 8 considered high. The sten scales are bipolar, meaning that each end of the scale has a distinct definition and meaning (Table - 1). Because bipolar scales are designated with "high" or "low" for each factor, a high score should not be considered to reflect a positive personality characteristic and a low score should not be considered to reflect a negative personality characteristic.

Table - 1 Description of personality traits measured by the 16 Personality Factors Questionnaires:

\begin{tabular}{|c|c|c|c|}
\hline $\begin{array}{l}\text { Sl. } \\
\text { No }\end{array}$ & Description of low range & Primary factors & Description of high range \\
\hline 1 & $\begin{array}{l}\text { Impersonal, distant, cool, } \\
\text { reserved, detached, formal, } \\
\text { aloof. }\end{array}$ & Warmth & $\begin{array}{l}\text { Warm, outgoing, attentive to } \\
\text { others, kindly, easygoing, } \\
\text { participating, likes people }\end{array}$ \\
\hline 2 & $\begin{array}{l}\text { Concrete-thinking, less } \\
\text { intelligent, lower general } \\
\text { mental capacity, unable to } \\
\text { handle abstract problems. }\end{array}$ & B- Reasoning & $\begin{array}{l}\text { Abstract-thinking, more } \\
\text { intelligent, bright, higher general } \\
\text { mental capacity, fast-learner. }\end{array}$ \\
\hline 3 & $\begin{array}{l}\text { Reactive emotionally, } \\
\text { changeable, affected by } \\
\text { feelings, emotionally less } \\
\text { stable, easily upset. }\end{array}$ & $\begin{array}{l}\text { C - Emotional } \\
\text { Stability. }\end{array}$ & $\begin{array}{l}\text { Emotionally stable, adaptive, } \\
\text { mature, faces reality calmly. }\end{array}$ \\
\hline 4 & $\begin{array}{l}\text { Deferential, cooperative, } \\
\text { avoids conflict, submissive, } \\
\text { humble, obedient, easily led, } \\
\text { docile, accommodating }\end{array}$ & E-Dominance & $\begin{array}{l}\text { Dominant, forceful, assertive, } \\
\text { aggressive, competitive, } \\
\text { stubborn, bossy }\end{array}$ \\
\hline 5 & $\begin{array}{l}\text { Serious, restrained, prudent, } \\
\text { taciturn, introspective, silent }\end{array}$ & F-Liveliness & $\begin{array}{l}\text { Lively, animated, spontaneous, } \\
\text { enthusiastic, happy-go-lucky, } \\
\text { cheerful, expressive, impulsive }\end{array}$ \\
\hline 6 & $\begin{array}{l}\text { Expedient, nonconforming, } \\
\text { disregards rules, self-indulgent }\end{array}$ & $\begin{array}{l}\text { G- Rule- } \\
\text { Consciousness }\end{array}$ & $\begin{array}{l}\text { Rule-conscious, dutiful, } \\
\text { conscientious, conforming, } \\
\text { moralistic, staid, rule-bound }\end{array}$ \\
\hline 7 & $\begin{array}{l}\text { Shy, threat-sensitive, timid, } \\
\text { hesitant, intimidated }\end{array}$ & H- Social Boldness & $\begin{array}{l}\text { Socially bold, venturesome, } \\
\text { thick-skinned, uninhibited }\end{array}$ \\
\hline 8 & $\begin{array}{l}\text { Utilitarian, objective, } \\
\text { unsentimental, tough-minded, } \\
\text { self-reliant, no-nonsense, } \\
\text { rough }\end{array}$ & I - Sensitivity & $\begin{array}{l}\text { Sensitive, aesthetic, sentimental, } \\
\text { tender-minded, intuitive, refined }\end{array}$ \\
\hline 9 & $\begin{array}{l}\text { Trusting, unsuspecting, } \\
\text { accepting, unconditional, easy }\end{array}$ & L - Vigilance & $\begin{array}{l}\text { Vigilant, suspicious, skeptical, } \\
\text { distrustful, oppositional }\end{array}$ \\
\hline 10 & $\begin{array}{l}\text { Grounded, practical, prosaic, } \\
\text { solution oriented, steady, } \\
\text { conventional }\end{array}$ & M - Abstractedness & $\begin{array}{l}\text { Abstract, imaginative, } \\
\text { absentminded, impractical, } \\
\text { absorbed in ideas }\end{array}$ \\
\hline 11 & $\begin{array}{l}\text { Forthright, genuine, artless, } \\
\text { open, guileless, naive, }\end{array}$ & $\mathrm{N}$ - Privateness & $\begin{array}{l}\text { Private, discreet, non-disclosing, } \\
\text { shrewd, polished, worldly, }\end{array}$ \\
\hline
\end{tabular}


Personality Traits of Parents and Problems Behaviors of the Children with Intellectual Disabilities

\begin{tabular}{|c|c|c|c|}
\hline $\begin{array}{l}\text { Sl. } \\
\text { No }\end{array}$ & Description of low range & Primary factors & Description of high range \\
\hline & unpretentious, involved & & astute, diplomatic \\
\hline 12 & $\begin{array}{l}\text { Self-assured, unworried, } \\
\text { complacent, secure, free of } \\
\text { guilt, confident, self-satisfied }\end{array}$ & $\mathrm{O}$ - Apprehension & $\begin{array}{l}\text { Apprehensive, self-doubting, } \\
\text { worried, guilt-prone, insecure, } \\
\text { worrying, self-blaming }\end{array}$ \\
\hline 13 & $\begin{array}{l}\text { Traditional, attached to } \\
\text { familiar, conservative, } \\
\text { respecting traditional ideas }\end{array}$ & $\begin{array}{l}\text { Q1 - Openness to } \\
\text { Change }\end{array}$ & $\begin{array}{l}\text { Open to change, experimental, } \\
\text { liberal, analytical, critical, } \\
\text { freethinking, flexibility }\end{array}$ \\
\hline 14 & $\begin{array}{l}\text { Group-oriented, affiliative, a } \\
\text { joiner and follower dependent }\end{array}$ & Q2 - Self-Reliance & $\begin{array}{l}\text { Self-reliant, solitary, resourceful, } \\
\text { individualistic, self-sufficient }\end{array}$ \\
\hline 15 & $\begin{array}{l}\text { Tolerates disorder, unexacting, } \\
\text { flexible, undisciplined, lax, } \\
\text { self-conflict, impulsive, } \\
\text { careless of social rules, } \\
\text { uncontrolled }\end{array}$ & Q3 - Perfectionism & $\begin{array}{l}\text { Perfectionist, organized, } \\
\text { compulsive, self-disciplined, } \\
\text { socially precise, exacting will } \\
\text { power, control, self-sentimental }\end{array}$ \\
\hline 16 & $\begin{array}{l}\text { Relaxed, placid, tranquil, } \\
\text { torpid, patient, composed low } \\
\text { drive }\end{array}$ & Q4 - Tension & $\begin{array}{l}\text { Tense, high-energy, impatient, } \\
\text { driven, frustrated, over-wrought, } \\
\text { time-driven }\end{array}$ \\
\hline
\end{tabular}

\section{Procedure of Data Collection}

The procedure for data collection involved use of cross-sectional individualized testing procedure for each child and their parents included as sample in this study. Only after the diagnosis was confirmed on the basis of clinical evaluation and psychological testing, the samples were chosen.

Interview of each parent was conducted in a quiet atmosphere with adequate privacy. At the beginning of interview a brief history regarding the demographic variable were taken on NIMH- SES scale. Then, problem behaviors of each child were assessed on BASIC-MR (PART-B) scale and severities of problem behaviors were calculated. For measuring the personality traits of parents, Sixteen Personality Factor questionnaire (Cattell, 1967) was administered. Form A of 16 PF personality test was used. Depending up on the educational background and comfort ability of the parents in the language use, both English and Hindi version of the questionnaire was used to assess their personality traits. The questionnaire contains 187 questions under 16 domains of personality traits. Parents were classified on ten point sten score for each personality factor in three groups, that is low score(1-3),Average (4-7) and High (8-10).

Table - 2 Description of the sample on socio-demographic variable

\begin{tabular}{|l|l|l|l|}
\hline Socio-demographic variable & Domain & $\mathbf{N}$ & Percentage (\%) \\
\hline \multirow{2}{*}{ Age } & Below 6 years of age & 05 & 11.12 \\
\cline { 2 - 4 } & 6-18 years of age. & 40 & 88.88 \\
\hline \multirow{3}{*}{ Gender of the child } & Male & 20 & 44.45 \\
\cline { 2 - 4 } & Female & 25 & 55.55 \\
\hline \multirow{3}{*}{ Bender of the parents } & Father & 27 & 60 \\
\cline { 2 - 4 } & Mother & 18 & 40 \\
\hline \multirow{3}{*}{ Background of the parents } & Urban & 34 & 75.56 \\
\cline { 2 - 4 } & Rural & 11 & 24.44 \\
\hline \multirow{5}{*}{ Parent's age } & $20-30$ years & 4 & 8.89 \\
\cline { 2 - 4 } & $31-40$ years & 29 & 64.44 \\
\cline { 2 - 4 } & $41-50$ years & 8 & 17.78 \\
\cline { 2 - 4 } & $51-60$ years & 4 & 8.89 \\
\hline
\end{tabular}

(C) The International Journal of Indian Psychology, ISSN 2348-5396 (e)| ISSN: 2349-3429 (p) | 6 
Personality Traits of Parents and Problems Behaviors of the Children with Intellectual Disabilities

\begin{tabular}{|l|l|l|l|}
\hline Socio-demographic variable & Domain & N & Percentage (\%) \\
\hline \multirow{3}{*}{ Socio-economic status (SES) $*$} & High & 03 & 6.67 \\
\cline { 2 - 4 } & Middle & 23 & 51.12 \\
\cline { 2 - 4 } & Low & 19 & 42.23 \\
\hline \multirow{5}{*}{ Educational level of the parents } & Below high school or X & 12 & 26.67 \\
\cline { 2 - 4 } & Class X to Graduation & 08 & 17.78 \\
\cline { 2 - 4 } & Up to post-graduation & 14 & 31.11 \\
\cline { 2 - 4 } & Post-graduation and above. & 11 & 24.44 \\
\hline
\end{tabular}

*Criteria of high-income group $=$ monthly income more than Rs. 10001, Middle income group $=$ monthly income in the range of Rs. 6501 to 10,000.00, Low income group $=$ monthly income less than Rs. 6500.00

\section{RESULT}

Table - 3. Description of the severity of Problem behavior as measured on BASIC - MR Part $-B$.

\begin{tabular}{|c|c|c|c|c|c|c|c|c|}
\hline $\begin{array}{l}\text { Types of } \\
\text { problem } \\
\text { behavior }\end{array}$ & $\begin{array}{l}\text { Levels of } \\
\text { ID }\end{array}$ & 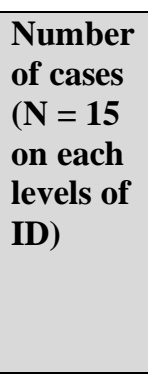 & $\begin{array}{l}\text { Score on } \\
\text { BASIC } \\
\text { - MR } \\
\text { part - } \\
\text { B. } \\
\text { (Max. } \\
\text { Possible } \\
\text { score= } \\
\text { 150). }\end{array}$ & Mean & SD & $\begin{array}{l}\text { Standard } \\
\text { error }\end{array}$ & $\mathbf{F}$ & $\begin{array}{l}\text { p- } \\
\text { value }\end{array}$ \\
\hline \multirow{3}{*}{$\begin{array}{l}\text { Violent and } \\
\text { destructive } \\
\text { Behavior. }\end{array}$} & Mild ID & 13 & 80 & 6.15 & 1.72 & 0.47 & \multirow[t]{3}{*}{$13.17 *$} & \multirow[t]{3}{*}{0.0001} \\
\hline & $\begin{array}{l}\text { Moderate } \\
\text { ID }\end{array}$ & 13 & 137 & 10.53 & 1.94 & 0.53 & & \\
\hline & Severe ID & 12 & 106 & 8.84 & 2.82 & 0.81 & & \\
\hline \multirow{3}{*}{$\begin{array}{l}\text { Temper } \\
\text { tantrum }\end{array}$} & Mild ID & 07 & 11 & 1.57 & 0.53 & 0.20 & \multirow[t]{3}{*}{$4.67 * *$} & \multirow[t]{3}{*}{0.018} \\
\hline & $\begin{array}{l}\text { Moderate } \\
\text { ID }\end{array}$ & 11 & 31 & 2.81 & 1.07 & 0.32 & & \\
\hline & Severe ID & 12 & 35 & 2.91 & 1.08 & 0.31 & & \\
\hline \multirow{3}{*}{$\begin{array}{l}\text { Misbehavior } \\
\text { with others }\end{array}$} & Mild ID & 13 & 44 & 3.38 & 1.98 & 0.54 & \multirow{3}{*}{$1.88 \#$} & \multirow{3}{*}{0.16} \\
\hline & $\begin{array}{l}\text { Moderate } \\
\text { ID }\end{array}$ & 13 & 53 & 4.07 & 1.03 & 0.28 & & \\
\hline & Severe ID & 13 & 39 & 3.00 & 1.08 & 0.29 & & \\
\hline \multirow{3}{*}{$\begin{array}{l}\text { Self-injurious } \\
\text { behavior }\end{array}$} & Mild ID & 12 & 30 & 2.5 & 0.79 & 0.23 & \multirow[t]{3}{*}{$10.41^{*}$} & \multirow[t]{3}{*}{0.0003} \\
\hline & $\begin{array}{l}\text { Moderate } \\
\text { ID }\end{array}$ & 13 & 45 & 3.46 & 0.96 & 0.26 & & \\
\hline & Severe ID & 12 & 58 & 4.84 & 1.80 & 0.51 & & \\
\hline \multirow{3}{*}{$\begin{array}{l}\text { Repetitive } \\
\text { behavior }\end{array}$} & Mild ID & 12 & 33 & 2.75 & 1.05 & 0.30 & \multirow[t]{3}{*}{$6.69^{*}$} & \multirow[t]{3}{*}{0.003} \\
\hline & $\begin{array}{l}\text { Moderate } \\
\text { ID }\end{array}$ & 11 & 43 & 3.90 & 1.26 & 0.38 & & \\
\hline & Severe ID & 13 & 57 & 4.38 & 1.65 & 0.47 & & \\
\hline \multirow[t]{3}{*}{ Odd behavior } & Mild ID & 13 & 51 & 3.92 & 1.40 & 0.38 & \multirow{3}{*}{$0.46 \#$} & \multirow{3}{*}{0.63} \\
\hline & $\begin{array}{l}\text { Moderate } \\
\text { ID }\end{array}$ & 14 & 50 & 3.57 & 1.22 & 0.32 & & \\
\hline & Severe ID & 13 & 44 & 3.38 & 1.04 & 0.28 & & \\
\hline \multirow{3}{*}{$\begin{array}{l}\text { Hyperactive } \\
\text { behavior }\end{array}$} & Mild ID & 11 & 45 & 4.09 & 1.13 & 0.34 & \multirow[t]{3}{*}{$0.48 \#$} & \multirow[t]{3}{*}{0.61} \\
\hline & $\begin{array}{l}\text { Moderate } \\
\text { ID }\end{array}$ & 12 & 53 & 4.41 & 1.56 & 0.45 & & \\
\hline & Severe ID & 12 & 47 & 3.91 & 0.99 & 0.28 & & \\
\hline \multirow{3}{*}{$\begin{array}{l}\text { Rebellious } \\
\text { behavior }\end{array}$} & Mild ID & 11 & 23 & 2.09 & 0.30 & 0.09 & \multirow[t]{3}{*}{$3.62 * *$} & \multirow[t]{3}{*}{0.03} \\
\hline & $\begin{array}{l}\text { Moderate } \\
\text { ID }\end{array}$ & 11 & 24 & 2.18 & 0.40 & 0.12 & & \\
\hline & Severe ID & 11 & 28 & 2.54 & 0.52 & 0.15 & & \\
\hline
\end{tabular}

(C) The International Journal of Indian Psychology, ISSN 2348-5396 (e)| ISSN: 2349-3429 (p) | 7 


\begin{tabular}{|c|c|c|c|c|c|c|c|c|}
\hline $\begin{array}{l}\text { Types of } \\
\text { problem } \\
\text { behavior }\end{array}$ & $\begin{array}{l}\text { Levels of } \\
\text { ID }\end{array}$ & 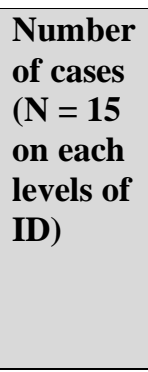 & $\begin{array}{l}\text { Score on } \\
\text { BASIC } \\
\text { - MR } \\
\text { part - } \\
\text { B. } \\
\text { (Max. } \\
\text { Possible } \\
\text { score= } \\
\text { 150). }\end{array}$ & Mean & SD & $\begin{array}{l}\text { Standard } \\
\text { error }\end{array}$ & $\mathbf{F}$ & $\begin{array}{l}\text { p- } \\
\text { value }\end{array}$ \\
\hline \multirow{3}{*}{$\begin{array}{l}\text { Antisocial } \\
\text { behavior }\end{array}$} & Mild ID & 05 & 09 & 1.8 & 0.44 & 0.2 & \multirow[t]{3}{*}{ 2.33\# } & \multirow[t]{3}{*}{0.12} \\
\hline & $\begin{array}{l}\text { Moderate } \\
\text { ID }\end{array}$ & 05 & 13 & 2.6 & 1.51 & 0.67 & & \\
\hline & Severe ID & 09 & 14 & 1.56 & 0.52 & 0.17 & & \\
\hline \multirow[t]{3}{*}{ Fear } & Mild ID & 08 & 14 & 1.75 & 0.46 & 0.16 & \multirow[t]{3}{*}{$2.25 \#$} & \multirow[t]{3}{*}{0.12} \\
\hline & $\begin{array}{l}\text { Moderate } \\
\text { ID }\end{array}$ & 09 & 18 & 2.00 & 0.70 & 0.23 & & \\
\hline & Severe ID & 11 & 26 & 2.36 & 0.67 & 0.20 & & \\
\hline
\end{tabular}

*Significant at 0.01 levels, ** Significant at 0.05 levels. , \# Not found significant.

Table - 4 Personality traits of the parents of children with ID - as measured on $16 \mathrm{PF}$.

\begin{tabular}{|c|c|c|c|c|c|c|}
\hline $\begin{array}{l}\text { Personality } \\
\text { traits on } 16 \text { PF }\end{array}$ & Levels & Mild ID & Moderate ID & Severe ID & $\begin{array}{l}\text { Chi- } \\
\text { square }\end{array}$ & $\mathbf{p}$ \\
\hline \multirow{3}{*}{$\begin{array}{l}\text { A } \\
\text { Warmth }\end{array}$} & Low & $1(6.67)$ & $4(26.67)$ & $2(13.34)$ & \multirow{3}{*}{3.25} & \multirow[t]{3}{*}{0.516} \\
\hline & Average & $12(80)$ & $10(66.67)$ & $10(66.67)$ & & \\
\hline & High & $2(13.34)$ & $1(6.67)$ & $3(20)$ & & \\
\hline \multirow{3}{*}{$\begin{array}{l}\text { B } \\
\text { Reasoning }\end{array}$} & Low & $0(0)$ & $0(0)$ & $0(0)$ & \multirow[t]{3}{*}{4.61} & \multirow[t]{3}{*}{0.329} \\
\hline & Average & $15(100)$ & $11(73.34)$ & 13 986.67) & & \\
\hline & High & $0(0)$ & $4(26.67)$ & $2(13.34)$ & & \\
\hline \multirow{3}{*}{$\begin{array}{l}\mathrm{C} \\
\text { Emotional } \\
\text { Stability }\end{array}$} & Low & $3(20)$ & $2(13.34)$ & $4(26.67)$ & \multirow[t]{3}{*}{3.06} & \multirow[t]{3}{*}{0.547} \\
\hline & Average & $12(80)$ & $13(86.67)$ & $10(66.67)$ & & \\
\hline & High & $0(0)$ & $0(0)$ & $1(6.67)$ & & \\
\hline \multirow{3}{*}{$\begin{array}{l}\text { E } \\
\text { Dominance }\end{array}$} & Low & $3(20)$ & $1(6.67)$ & $1(6.67)$ & \multirow[t]{3}{*}{7.97} & \multirow[t]{3}{*}{0.092} \\
\hline & Average & $12(80)$ & $11(73.34)$ & $14(93.34)$ & & \\
\hline & High & $0(0)$ & $3(20)$ & $0(0)$ & & \\
\hline \multirow{3}{*}{$\begin{array}{l}\mathrm{F} \\
\text { Liveliness }\end{array}$} & Low & $8(53.34)$ & $4(26.67)$ & $2(13.34)$ & \multirow[t]{3}{*}{5.08} & \multirow[t]{3}{*}{0.279} \\
\hline & Average & $7(46.67)$ & $11(73.34)$ & $13(86.67)$ & & \\
\hline & High & $0(0)$ & $0(0)$ & $0(0)$ & & \\
\hline \multirow{3}{*}{$\begin{array}{l}\text { G } \\
\text { Rule- } \\
\text { consciousness }\end{array}$} & Low & $0(0)$ & $3(20)$ & $1(6.67)$ & \multirow[t]{3}{*}{7.65} & \multirow[t]{3}{*}{0.105} \\
\hline & Average & $13(86.67)$ & $12(80)$ & $14(93.34)$ & & \\
\hline & High & $2(13.34)$ & $0(0)$ & $0(0)$ & & \\
\hline \multirow{3}{*}{$\begin{array}{l}\mathrm{H} \\
\text { Social boldness }\end{array}$} & Low & $2(13.34)$ & $0(0)$ & $2(13.34)$ & \multirow[t]{3}{*}{4.35} & \multirow[t]{3}{*}{0.360} \\
\hline & Average & $12(80)$ & $15(100)$ & $13(86.67)$ & & \\
\hline & High & $1(6.67)$ & $0(0)$ & $0(0)$ & & \\
\hline \multirow{3}{*}{ I } & Low & $2(13.34)$ & $0(0)$ & $3(20)$ & \multirow[t]{3}{*}{$9.82 *$} & \multirow[t]{3}{*}{0.043} \\
\hline & Average & $10(66.67)$ & $15(100)$ & $12(80)$ & & \\
\hline & High & $3(20)$ & $0(0)$ & $0(0)$ & & \\
\hline \multirow{3}{*}{$\begin{array}{l}\mathrm{L} \\
\text { Vigilance }\end{array}$} & Low & $1(6.67)$ & $0(0)$ & $0(0)$ & \multirow[t]{3}{*}{6.25} & \multirow[t]{3}{*}{0.181} \\
\hline & Average & $9(60)$ & $5(33.34)$ & $10(66.67)$ & & \\
\hline & High & $5(33.34)$ & $10(66.67)$ & $5(33.34)$ & & \\
\hline \multirow{3}{*}{$\begin{array}{l}\text { M } \\
\text { Abstractedness }\end{array}$} & Low & $5(33.34)$ & $5(33.34)$ & $0(0)$ & 6.42 & 0.169 \\
\hline & Average & $10(66.67)$ & $10(66.67)$ & $15(100)$ & & \\
\hline & High & $0(0)$ & $0(0)$ & $0(0)$ & & \\
\hline $\mathrm{N}$ & Low & $1(6.67)$ & $1(6.67)$ & $0(0)$ & 2.94 & 0.567 \\
\hline Privateness & Average & $9(60)$ & $12(80)$ & $12(80)$ & & \\
\hline & High & $5(33.34)$ & $2(13.34)$ & $3(20)$ & & \\
\hline $\mathrm{O}$ & Low & $0(0)$ & $0(0)$ & $0(0)$ & 3.84 & 0.428 \\
\hline Apprehension & Average & 14 (93.34) & $15(100)$ & $12(80)$ & & \\
\hline
\end{tabular}


Personality Traits of Parents and Problems Behaviors of the Children with Intellectual Disabilities

\begin{tabular}{|c|c|c|c|c|c|c|}
\hline $\begin{array}{l}\text { Personality } \\
\text { traits on } 16 \text { PF }\end{array}$ & Levels & Mild ID & Moderate ID & Severe ID & $\begin{array}{l}\text { Chi- } \\
\text { square }\end{array}$ & $\mathbf{p}$ \\
\hline & High & $1(6.67)$ & $0(0)$ & $3(20)$ & & \\
\hline \multirow{3}{*}{$\begin{array}{l}\text { Q1 } \\
\text { Openness to } \\
\text { change }\end{array}$} & Low & $1(6.67)$ & $1(6.67)$ & $1(6.67)$ & \multirow[t]{3}{*}{ 11.58* } & \multirow[t]{3}{*}{0.020} \\
\hline & Average & $11(73.34)$ & $5(33.34)$ & $13(86.67)$ & & \\
\hline & High & $3(20)$ & $9(60)$ & $1(6.67)$ & & \\
\hline \multirow{3}{*}{$\begin{array}{l}\text { Q2 } \\
\text { Self-reliance }\end{array}$} & Low & $0(0)$ & $2(13.34)$ & $2(13.34)$ & \multirow[t]{3}{*}{5.66} & \multirow[t]{3}{*}{0.226} \\
\hline & Average & $12(80)$ & $12(80)$ & $8(53.34)$ & & \\
\hline & High & $3(20)$ & $1(6.67)$ & $5(33.34)$ & & \\
\hline \multirow{3}{*}{$\begin{array}{l}\text { Q3 } \\
\text { Perfectionism }\end{array}$} & Low & $3(20)$ & $3(20)$ & $1(6.67)$ & \multirow[t]{3}{*}{3.37} & \multirow[t]{3}{*}{0.497} \\
\hline & Average & $10(66.67)$ & $12(80)$ & $12(80)$ & & \\
\hline & High & $2(13.34)$ & $0(0)$ & $2(13.34)$ & & \\
\hline \multirow{3}{*}{$\begin{array}{l}\text { Q4 } \\
\text { Tension }\end{array}$} & Low & $0(0)$ & $1(6.67)$ & $2(13.34)$ & \multirow[t]{3}{*}{2.14} & \multirow[t]{3}{*}{0.710} \\
\hline & Average & $15(100)$ & $14(93.34)$ & $13(86.67)$ & & \\
\hline & High & $0(0)$ & $0(0)$ & $0(0)$ & & \\
\hline
\end{tabular}

Note: Fingers in parentheses indicates percentage. *Significant at 0.05 level

\section{DISCUSSION}

Description of the personality traits as measured by $16 \mathrm{PF}$ questionnaires were given in table-1. Cattell, 1967 described 16 primary factors of personality traits that were indicated in alphabetical order of A, B, C, E, F, G, H, I, L, M, N, O, Q1, Q2, Q3 and Q4. He has named all of these factors as warmth $(\mathrm{A})$, reasoning $(\mathrm{B})$, emotional stability $(\mathrm{C})$, dominance $(\mathrm{E})$, liveliness $(\mathrm{F})$, rule-consciousness $(\mathrm{G})$, social-boldness $(\mathrm{H})$, sensitivity $(\mathrm{I})$, vigilance $(\mathrm{L})$, abstractedness $(\mathrm{M})$, privateness $(\mathrm{N})$, apprehension $(\mathrm{O})$, openness to change $(\mathrm{Q} 1)$, selfreliance $(\mathrm{Q} 2)$, perfectionism $(\mathrm{Q} 3)$ and tension $(\mathrm{Q} 4)$. Score in the range of 4 to 7 is considering the average, balanced response that is expected from a normal and mentally healthy person. Score in the range of 8 to 10 is consider as a high score and score in the range of 1 to 3 is consider the very low score. Both of these scores (high and low) is consider the deviation of the scores and generally not consider good for the healthy development of the personality. Description of both of the range of scores (low range and high range) of all 16 personality factors is given in table-1.

The description of the sample on different socio-demographic variables has been presented in table-2. This table indicates that most of the cases were belongs to in the age range of 6 18 years $(\mathrm{N}=40,88.88 \%)$ among them most of them were females $(\mathrm{N}=25,55.55 \%)$. The parents who were interviewed on the dimensions of problem behaviors showed by their children and subsequent assessment of the personality traits, most of them were father $(\mathrm{N}=$ $27,60 \%)$, who belongs to urban background $(\mathrm{N}=34,75.56 \%)$. Most of the informant were in the age range of $31-40$ years $(\mathrm{N}=29,64.44 \%)$ followed by $41-50$ years $(\mathrm{N}=8,17.78 \%)$. Those parents who were very young (21-30 years) or very elderly (51-60 years) were very less $(\mathrm{N}=4,8.89 \%$ respectively on each group).

Most of the informant belongs to middle socio-economic status $(\mathrm{N}=23,51.12 \%)$ followed by low socio-economic status $(\mathrm{N}=19,42.23 \%)$. Those who belongs to high socio-economic status were relatively less $(\mathrm{N}=3,6.67 \%)$. The criteria used to distribute the sample into three categories (High, middle and low socio-economic status) is based on their per month income. Those who were earning Rs. 6500.00 or less per month from all sources were kept under low socio-economic status. Those who are earning per month in the range of Rs. 6501 to $10,000.00$ from all sources were kept under middle socio-economic status. Those monthly income is Rs. 10,001 or above were kept under the category of high socio-economic status. Most of the informant were educated up to post-graduation $(\mathrm{N}=14,31.11 \%)$ followed by educated up to below high school or Xth class $(\mathrm{N}=12,26.67 \%)$. Those who were educated

(c) The International Journal of Indian Psychology, ISSN 2348-5396 (e)| ISSN: 2349-3429 (p) | 9 
up to graduation or more than post-graduation were relatively low in number $(\mathrm{N}=8,17.78 \%$ and $\mathrm{N}=11,24.44 \%$ respectively on each group).

In table -3 , description of the problem behavior as measured on BASIC- MR, Part - B - is presented. This table indicates that most of the mildly, moderately and severely intellectually disabled children showed violent and destructive behavior $(\mathrm{F}=13.17$, significant at 0.01 level) followed by self-injurious behavior $(\mathrm{F}=10.41$, significant at 0.01 level $)$ and repetitive behavior $(\mathrm{F}=6.69$, significant at 0.01 level). Temper tantrum behavior and rebellious behavior was also found in the children that were significant at 0.05 level $(\mathrm{F}=4.67$ and $\mathrm{F}=$ 3.62 respectively). Hence, the null hypothesis 1 is rejected that states that there is no significant difference in problem behavior exhibited by children with different levels of ID. That means the alternative hypothesis is accepted that states that there is a difference in the exhibition of problem behavior by the children with different levels of ID. All three groups of children with ID are homogenous in the terms of exhibition of problem behavior. Those problem behaviors which was not found significant are misbehavior with others $(\mathrm{F}=1.88)$, odd behavior $(\mathrm{F}=0.46)$, hyperactive behavior $(\mathrm{F}=0.48)$, antisocial behavior $(\mathrm{F}=2.33)$, and fears $(\mathrm{F}=2.25)$.

In the table -4 , data related to the personality traits of the parents as measured on $16 \mathrm{PF}$ questionnaires were presented. Only those parents were included in the study who have children with ID and must exhibiting problem behavior in one or another area/s of BASIC MR, Part-B. Chi-square test was computed on different traits of personality as measured on $16 \mathrm{PF}$ test. This table indicates that only two factors, that is factor I (sensitivity) and factor Q1 (openness to change) were significant at 0.05 levels (chi-square $=9.82$ and 11.58 respectively) that means children of those parents who obtained high score on the personality traits of $\mathrm{I}$ and Q1 were involve more in problem behavior. Hence, null hypothesis -2 is also rejected, that tells us there is no significant difference in personality traits of the parents having children with different levels of ID. That means the alternative hypothesis is accepted that states there is difference in personality traits of parents having children with different levels of ID. Only those children exhibited more problem behaviors whose parents holds the factors I and Q1 in their personality traits in high amount.

To elaborate it we can say that those parents who scored high on factor I (sensitivity, aesthetic, sentimental, tender-minded, intuitive and redefined) were showed more concern about their children with ID and such children were more prone to exhibit problem behavior. Similarly, those parents who were scored high on factor Q1 (openness to change, experimental, liberal, analytical, critical, free-thinking, and flexible) were also showed their more concern about their children with ID and such children were more prone to exhibit problem behavior.

Remaining factors of 16 PF were not found significant. Hence it is difficult to say anything with full certainty about its existence with problem behavior.

\section{CONCLUSION}

It has been found that violent and destructive behavior, self-injurious behavior, repetitive behavior, temper tantrum behavior and rebellious behavior was present in ascending order in all three groups of children with ID (Mild, moderate and severe levels of ID).

The children of those parents who obtained high score on the personality traits of I (Sensitivity) and Q1 (Openness to change) were involve more in problem behavior. 


\section{Limitations}

The present study is having some limitations that one should take into account when interpreting the results. First, both parents were not included in this study, either father or mother's personality was taken to see the relation with problem behavior of their children. This is not giving complete picture of impact of parent's personality traits on problem behavior in their children with intellectual disability. Secondly, sample size was small and taken from specific cluster, so its finding cannot be generalized. A third limitation of the present study is other variables (such as parent's living conditions, their economic and social background etc.) have also impact on problem behaviors of children with intellectual disabilities, which was not considered in this research.

\section{REFERENCES}

American Psychiatric Association. (1994). Diagnostic and statistical manual of mental disorders. (4th Ed.), Washington DC: American Psychiatric Association.

American Psychiatric Association. (2000). Diagnostic and statistical manual of mental disorders. (4th Ed.), Washington DC: American Psychiatric Association.

Barlow, D. H., \& Durand, V. M. (2009). Abnormal psychology: an integrative approach. (5 ed.). Belmont, CA: Wadsworth Cengage Learning.

Belsky, J., Hsieh, K.-H., \& Crnic, K. (1998). Mothering, fathering, and infant negativity as antecedents of boys' externalizing problems and inhibition at age 3 years: Differential susceptibility to rearing experience? Development and Psychopathology, 10, 301-319.

Burchinal, Lee. (1964). Characteristics of Adolescents from Unbroken and Broken Families. Journal of Marriage and Family, December, 44-51.

Carver, C. S., \& Scheier, M. F. (2000). Perspectives on personality (4th ed.) Boston: Allyn and Bacon.

Cattell, R.B. Handbook for the Sixteen Personality Factor Questionnaire. Champaign, Illinois: Institute for Personality and Ability Testing, 1967. Handbook Supplement for Form C.

Glassner,Paul and Elizabeth Navarre,(1965). Structural Problems of the One Parent Family. Journal of Social Issues, XXI, January,98-109.

McCarthy,Janet.(1968). Changing Parent Attitudes and Improving Intellectual Abilities of Culturally Disadvantaged Four Year Old Children Through Parent Involvement, U.S.Educational Resources Information Centre, E.R.I.C Document, ED 207-619.

NIMH. (1999). The NIMH Socio Economic Status Scale for case history taking. Secunderabad: National Institute for the Mentally Handicapped.

Paulous, Susan. (1969).A Problem Inventory of Single Mothers, Vancouver: Children's Aid Society.

Peniston,Eugene.(1976). An Evaluation of the Portage Project: A Comparison of a Home Visit Programme for Multiple Handicapped Pre-School and Headstart Programme, U.S Educational Resources Information Centre. E.R.I.C Document, ED 112-520

Peshawaria, R., \& Venkatesan, S (1992). Behavioural Approach in Teaching Mentally Retarded Children,Secunderabad ;National Institute for the Mentally Handicapped.

Peshawaria, R., \& Venkatesan, S., (1992). Behavioural Assessment Scales for Indian Children with Mental Retardation (BASIC-MR).Secunderabad: National Institute for the Mentally Handicapped.

Rastogi, C.K. (1984). Personality Pattern of Parents of mentally retarded children, Indian Journal of Psychiatry, 26, 46-50. 
The Rights of Persons with Disabilities Act, (2016). Gazette of India (Extra-ordinary); 28 December, 2016. Available from http://www.disabilityaffairs.gov.in/uploaad/uploadfiles/files/RPWD/ACT/2016.pdf .

Srivastava, R. K., Seth, M., \& Shobha, S. (1981). Maternal personality of educable mentally retarded children on Cattell's 16 Personality Factor test, Child Psychiatry Quarterly, 14,100-107.

\section{Acknowledgements}

The author appreciates all those who participated in the study and helped to facilitate the research process.

\section{Conflict of Interest}

The author declared no conflict of interest.

How to cite this article: A K Santosh \& J Prakash (2020). Personality Traits of Parents and Problems Behaviors of the Children with Intellectual Disabilities. International Journal of Indian Psychology, 8(3), 1-12. DIP:18.01.001/20200803, DOI:10.25215/0803.001 